\title{
A Case Study on Reviewing Specialist Services Commissioning in Wales: TAVI for Severe Aortic Stenosis
}

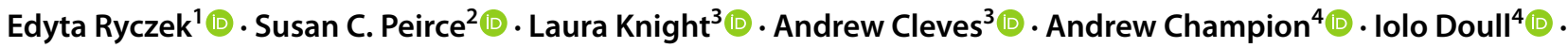 \\ Sian Lewis ${ }^{4}[$
}

Accepted: 21 October 2021 / Published online: 25 February 2022

(c) The Author(s) 2022

\begin{abstract}
The Welsh Health Specialised Services Committee (WHSSC) is responsible for planning, commissioning and funding specialised healthcare in Wales. Investment in new technologies or services is based on clinical and economic evidence, using a consistent and transparent process. This is accomplished in three stages. The first stage is the preparation of a rapid evidence review. This then informs the development or update of the relevant Commissioning Policy. The final stage is to prioritise the Commissioning Policy recommendations against all other new services and interventions, to inform WHSSC's annual commissioning intentions. In 2017, a review was conducted of the WHSSC Commissioning Policy for transcatheter aortic valve implantation for severe aortic stenosis. Prior to this only high-risk patients were eligible for transcatheter aortic valve implantation. The rapid evidence review identified three randomised controlled trials and two economic analyses relevant to the decision problem. Transcatheter aortic valve implantation was generally found to be more expensive and more effective than medical management or surgical aortic valve replacement, with incremental cost-effectiveness ratios around $£ 10,500-£ 36,000$ for inoperable groups and $£ 17,000-£ 24,000$ in high-risk groups. The rapid evidence review, expert advice and stakeholder feedback informed the revision process of the Commissioning Policy for transcatheter aortic valve implantation. This recommended the addition of patients unsuitable for surgical aortic valve replacement and the removal of explicit risk scoring. This recommendation was subject to the prioritisation process (carried out annually). The updated transcatheter aortic valve implantation recommendation was ranked second out of 23 technologies and services competing for additional WHSSC funding. The WHSSC Integrated Commissioning Plan for specialised services in Wales (2019) therefore included funding to support the new criteria for transcatheter aortic valve implantation treatment.
\end{abstract}

\section{Plain Language Summary}

In Wales, specialised health services are selected and funded at a national level by the Welsh Health Specialised Services Committee. Specialised services are provided for small numbers of patients, requiring highly specialised professionals or technologies. When the aortic heart valve becomes narrowed with disease it can be replaced with an artificial valve. This normally requires open surgery, which is risky for some patients, particularly those who are frail. Since 2012, the Welsh Health Specialised Services Committee have funded a less invasive procedure called TAVI (transcatheter aortic valve implantation) for patients who could have open surgery but at a high risk. In 2017, this policy needed updating, thus a new evidence review was conducted. This showed that patients at high risk from open surgery were more likely to survive if they

Susan C. Peirce

Peirces@cardiff.ac.uk

Laura Knight

Laura.knight2@wales.nhs.uk

Andrew Cleves

Andrew.cleves@wales.nhs.uk

Andrew Champion

Andrew.Champion@wales.nhs.uk

Iolo Doull

Iolo.Doull2@wales.nhs.uk
Sian Lewis

Sian.Lewis100@wales.nhs.uk

1 York, UK

2 Cedar, Cardiff Medicentre, Cardiff University, Heath Park, Cardiff CF14 4UJ, UK

3 Cedar, Cardiff and Vale University Health Board, Cardiff, UK

4 Welsh Health Specialised Services Committee, Pontypridd, UK 
underwent TAVI. Others, for whom open surgery was too risky, were also more likely to survive if they underwent TAVI instead of medication. However, TAVI tended to produce more vascular problems, such as blockages or damage to blood vessels. Transcatheter aortic valve implantation is generally more effective and more expensive than either drugs or open surgery in these patient groups, but is within cost-effectiveness limits often used in the UK National Health Service. As a result of the review, experts recommended that TAVI should be available to more patients, which would require greater levels of funding. Transcatheter aortic valve implantation was ranked as second out of 23 new or updated treatments competing for funding allocations. The Welsh Health Specialised Services Committee therefore published a new Commissioning Plan for TAVI in 2019 that now included patients who are considered too risky to undergo open surgery.

\section{Introduction}

Specialised healthcare services are those provided for rare and complex conditions that may require a critical mass of expertise and/or financial resources. They are often defined by the patient population, the number of providers that can deliver the service and the cost of providing it. In Wales, the Welsh Health Specialised Services Committee (WHSSC) is responsible for the planning and commissioning of specialised and tertiary services, enabling equitable access to safe, effective and sustainable provision within available resources [1,2]. Funding for WHSSC is primarily provided from the seven Welsh Health Boards to cover a total population of around 3 million people. The services are provided for Welsh residents, but some are carried out by healthcare organisations in England. In 2019-20, the WHSSC budget was $£ 632$ million, representing approximately $9.5 \%$ of the total spend for National Health Service (NHS) Wales Health and Social Care [3].

\subsection{Commissioning Specialised Services in Wales}

WHSSC annually produces an Integrated Commissioning Plan (ICP), which describes in detail the specialised services that it will commission with this budget for the following 3 years. The ICP includes provision for new treatments.

Innovation in specialised services often represents treatments of high cost for low patient numbers. National Health Service Wales and WHSSC must ensure that new investment decisions are (i) affordable and offer value for money; (ii) supported by convincing evidence of safety and effectiveness, and (iii) made using a process that is consistent and transparent. To achieve this, WHSSC has developed a process to compare competing proposals for new investment. Each year WHSSC carries out a prioritisation process to consider the commissioning of new interventions and technologies and the expansion of existing services.

The criteria that must be met for Welsh patients to access a treatment or service commissioned by WHSSC are defined by a Commissioning Policy (CP). This describes the service, drug or technology, the relevant clinical indications and which groups of patients are eligible. The WHSSC process for developing or updating a CP starts with a rapid review of the relevant clinical and economic evidence (stage 1, see Fig. 1). To ensure objectivity, WHSSC commissions Cedar, a healthcare technology research centre, to independently conduct the rapid evidence reviews (RERs). WHSSC also engages with clinical experts from across Wales and establishes an Expert Advisory Group (EAG) for each topic, who consider the evidence and propose any changes to the $\mathrm{CP}$ (stage 2). This evidence informs the WHSSC decision-making process: prioritisation against other new treatments and services, assessment of affordability and sign off by a Joint Committee of all NHS Wales Health Board chief executives (stage 3).

The annual WHSSC prioritisation process helps ensure the NHS in Wales commissions clinically and cost-effective services, and makes new treatments or services available in a timely manner. Prioritisation allows new interventions to be ranked according to a set of pre-determined criteria, including their clinical and cost effectiveness. This is combined with information on demands from existing services to underpin and feed into the development of the WHSSC annual ICP. Any widening of the access criteria proposed by the EAG is considered within this process.

The scoring and ranking of new treatments or services are carried out by a multidisciplinary Prioritisation Panel consisting of healthcare professionals, commissioners, legal experts and lay members. The Panel uses a standardised methodology, aiming for a fair and transparent process to ensure that evidence-based healthcare gain and value for money is maximised [4]. The process is specific for Wales and therefore reflects the needs and priorities of its population.

Members score each intervention against the following criteria to rank their relative priority:

- patient benefit (clinical impact);

- burden of disease - nature (severity) of the condition;

- burden of disease - population impact;

- potential for improving/reducing inequalities of access.

The aim of this paper is to describe the standard methodology used by WHSSC to update a CP, using the example of transcatheter aortic valve implantation (TAVI) for severe aortic stenosis (AS). We describe how recommended 
Fig. 1 Welsh Health Specialised Services Committee (WHSSC) process for updating or develop-

(CPs) ing Commissioning Policies

\section{$\mathrm{CP}$ (intervention) specific \\ processes \\ Annual generic processes}

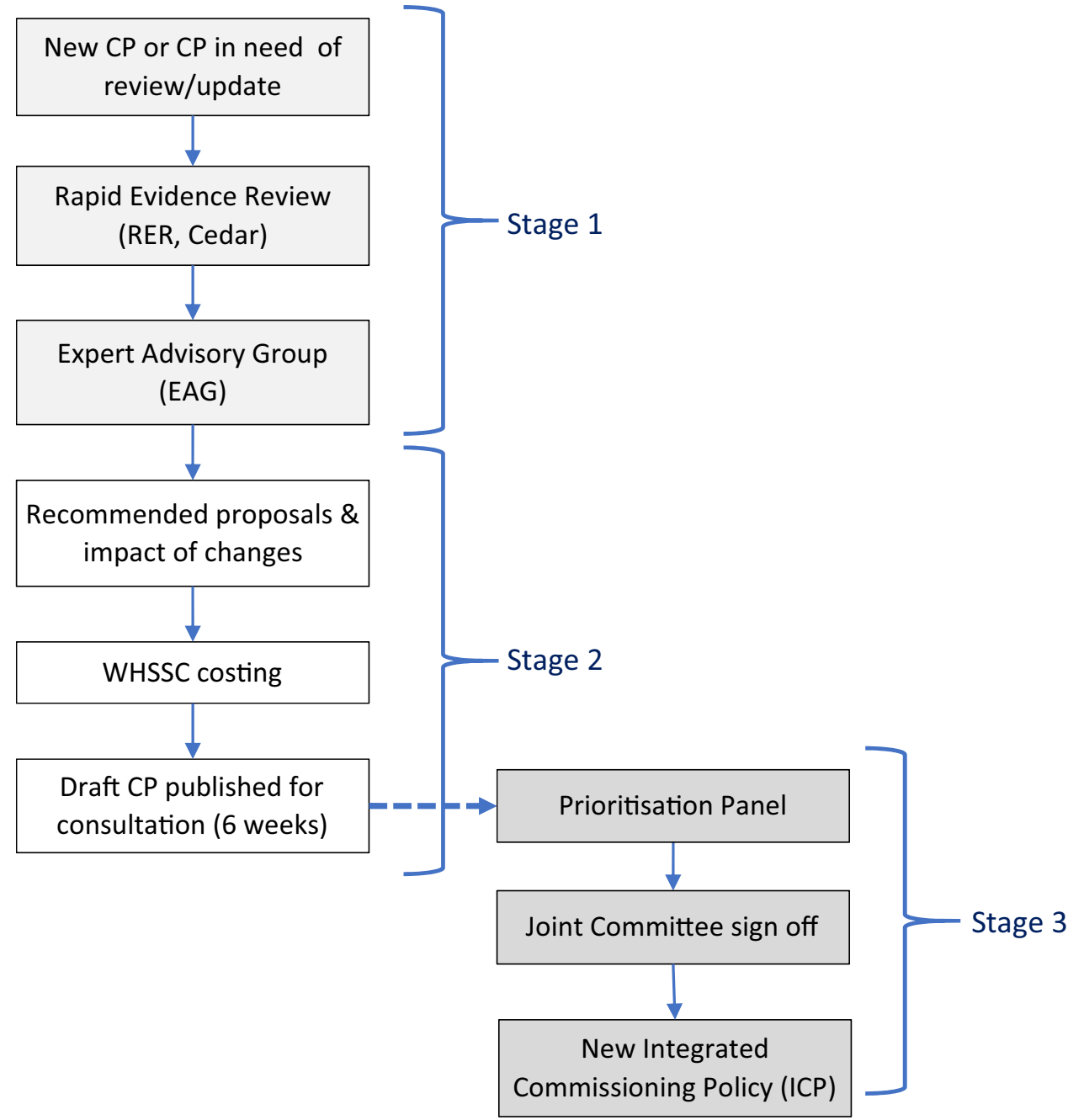

changes to the eligible patient population were prioritised against competing funding demands, and how TAVI was commissioned for the resident population of Wales.

\subsection{Aortic Stenosis (AS)}

Aortic stenosis (narrowing of the aortic valve) can manifest as angina pectoris, syncope or heart failure, and carries a poor prognosis. The prevalence of AS increases with age and affects between 2 and $5 \%$ of people aged 75 years and older $[5,6]$. Treatment options for patients with AS include medical management and surgical intervention, usually open surgical aortic valve replacement (SAVR). Surgical aortic valve replacement results in symptomatic relief, improved quality of life and long-term survival. However, SAVR requires sternotomy and cardiopulmonary bypass, and is associated with high perioperative morbidity and mortality in patients with advanced age, frailty and co-morbidities [6]. Thus a significant number of elderly patients considered at very high risk do not progress to SAVR, and with increasing longevity this population will rise over time [6].

The operative risk for patients with AS is assessed using validated scoring systems such as the Society of Thoracic Surgeons (STS) or European System for Cardiac Operative Risk Evaluation (EuroSCORE/EuroSCORE II) [8]. Transcatheter aortic valve implantation was developed as a less invasive alternative to SAVR and has become the treatment of choice for inoperable or high-risk patients with AS. An artificial valve is implanted using minimally invasive techniques, such as endovascular access via the femoral artery. 


\subsection{Background to the Transcatheter Aortic Valve Implantation (TAVI) Policy Update}

In 2017, the WHSSC TAVI CP (CP58) was considerably overdue for review [9]. It supported the use of TAVI in patients who:

- had been referred to surgery in the first instance;

- had a logistic EuroSCORE $>20 \%$ and STS score $>10 \%$;

- had a life expectancy of at least 12 months;

- and where the balance of risks and benefits favoured TAVI as judged by the multidisciplinary team.

Patients unsuitable for surgery because of technical reasons (e.g. "porcelain aorta" due to calcification) were considered for TAVI irrespective of EuroSCORE. This risk scoring means that patients were eligible for TAVI if they were at high surgical risk for SAVR.

There was consensus across the cardiac community in Wales that the policy no longer reflected current clinical practice and was inconsistent with most published guidance and the current evidence base. In September 2017, Cedar were commissioned to produce an RER on the clinical and cost effectiveness of TAVI in adults with heart failure secondary to AS for whom surgery is considered suitable but high risk. It was noted that the NHS England TAVI CP [10] included both high-risk and inoperable patients whereas CP58 only covered patients who are considered 'high surgical risks'.

\subsection{Extant UK Guidance}

In July 2017, the UK National Institute for Health and Care Excellence (NICE) published updated Interventional Procedures Guidance (IPG586) that concluded that evidence on the safety and efficacy of TAVI supported its routine use [11]. This was a change to the previous recommendations in IPG421 (2012), which recommended its routine use only in patients unsuitable for surgery, and use with special arrangements for patients with a high surgical risk [12].

NICE Clinical Guideline 187 for acute heart failure (2014) recommends TAVI in people who are assessed as unsuitable for SAVR [13]. Unlike IPG586, CG187 included economic evidence. Four UK-based economic cost-utility analyses were included in the evaluation; three comparing TAVI against medical management in patients deemed unsuitable for surgery [14-16] and one comparing TAVI with SAVR in high-risk patients [17]. In inoperable patients, TAVI was found to be more costly and more effective than medical management, with incremental cost-effectiveness ratios (ICERs) of $£ 12,900$ [15], £16,200 [16] and £35,956 per quality-adjusted life-year (QALY) [14] (at 2012 and 2013 prices). Despite calculating a lower increase in costs for TAVI than the other studies, Murphy et al. reported a higher ICER primarily because of much lower gains in QALYs [14]. In high-risk patients, TAVI was $£ 1350$ less expensive than SAVR and more effective (2013 prices) [16].

The 2017 Scottish Health Technologies Group Advice Statement 001/17 supports the provision of TAVI for adults with AS who are deemed to be at high surgical risk [18]. (Patients deemed not suitable for surgery were already provided with the option of TAVI at a single centre.) To inform this guidance, Healthcare Improvement Scotland evaluated the cost effectiveness of TAVI vs SAVR, reporting ICERs of $£ 17,064-£ 23,130$ in the high-risk group. A sensitivity analysis indicated that TAVI device costs and 2-year mortality rates were key drivers in the model [6].

All of these analyses used data from the PARTNER and/ or CoreValve studies (see Sect. 3 for study information). Concerns were noted regarding the generalisability of the risk profiles of the study samples to the general UK populations because of the selection criteria used.

\subsection{Extant International Guidance}

The joint European Society of Cardiology and European Association for Cardio-Thoracic Surgery 2017 guidelines on valvular heart disease recommend TAVI for patients unsuitable for surgery [19]. For patients with a high surgical risk, the choice of surgical modality is determined by the specialist Multidisciplinary Team (MDT), although TAVI is preferred in elderly patients suitable for transfemoral access. Surgical aortic valve replacement is recommended for low-risk patients. To counteract the limitations of STS, EuroSCORE II and logistic EuroSCORE risk scores, the guidelines include additional factors to be considered by the MDT (such as frailty, porcelain aorta, anatomy and co-morbidities).

The joint American Heart Association and American College of Cardiology guidelines on valvular heart disease recommend TAVI for patients with a prohibitive surgical risk [20]. For patients with high surgical risk, TAVI is a 'reasonable alternative' to SAVR, but SAVR is recommended in patients with low or intermediate surgical risk.

\section{Rapid Review Methods}

The aim of the WHSSC RER was to identify evidence for the clinical and cost effectiveness of TAVI in adults with heart failure secondary to AS, and for whom SAVR is considered unsuitable or high risk. A rapid review is systematic, but simplifies or omits one or more elements of a traditional systematic review in order to inform decisions in a timely manner [21]. A substantial systematic review had been performed in May 2017 to inform NICE 
guidance IPG568 [22]. We took a pragmatic approach and used this systematic review as the baseline for our RER. We updated the NICE clinical literature search to identify any randomised controlled trials (RCTs) published after this date. The NICE systematic review considered evidence for all surgical risk groups, but segregated this into inoperable, high risk and lower risk categories. NICE Interventional Procedures Guidance does not consider economic evidence, thus a separate search was conducted for new economic evidence from 2011 onwards.

A PICO (Population, Intervention, Comparator, Outcomes) framework was developed in consultation with a clinical advisor (Table 1). This was used to create search strategies run on the following databases on 10 October, 2017: MEDLINE, MEDLINE In-Process, Embase, Cochrane Library and PubMed ('Epub ahead of press'). This limited the search to RCTs published between May and October 2017. The economic search used the same basic search terms, but replaced RCT-related search terms with those that described economic studies. Economic evidence with a UK perspective was included if not already reviewed in UK guidance [14-17].

Records were screened by one reviewer at title and abstract stage, and full texts of the remaining papers were further screened for eligibility. Uncertainties were discussed with a second reviewer. The systematic review for IPG568 [22] assessed studies for bias; no additional quality appraisal was conducted for this RER. Two reviewers extracted and cross-checked key clinical and cost-effectiveness data into evidence tables. The classification of risk groups differed across studies, thus we detailed the study inclusion criteria, patients' baseline characteristics and health outcomes.

\section{Rapid Review Results}

Following removal of duplicates, 378 reports were identified via the clinical study update, and 216 reports from the economic search. No new RCTs that met the inclusion criteria were identified. Two UK-based cost-effectiveness studies were identified, using data from UK hospitals [23] or published international randomised studies [24].

To inform the WHSSC policy review, the studies on inoperable and high-risk patients included in NICE IPG568 were summarised. In total there were six RCTs in this guidance, reported in multiple publications. These six RCTs included patients from three distinct risk groups: inoperable (PARTNER 1B), high risk (PARTNER 1A, CoreValve) and intermediate/low risk (PARTNER 2A, NOTION, SURTAVI). The PARTNER 1B, PARTNER 1A and CoreValve studies met our inclusion criteria. We additionally used reference list searching to identify reports from these three RCTs that contained the maximum information about patient characteristics and the longest follow-up periods. This resulted in seven publications on three RCTs used as the clinical evidence for this RER. Table 2 summarises the RCTs and publications included in the RER.

Figure 2 shows the recruitment periods of the six studies included in IPG568 and illustrates that TAVI studies have progressed from inoperable/high-risk patients to lower risk groups over time. PARTNER 1A and 1B are subpopulations (high risk and inoperable, respectively) from the same overarching study.

\subsection{Clinical Evidence}

A summary of evidence is provided for inoperable and high surgical risk separately, with study details reported in
Table 1 PICO (Population, Intervention, Comparator, Outcomes) framework and inclusion/exclusion criteria for the rapid evidence review

\begin{tabular}{ll}
\hline Population & $\begin{array}{c}\text { Adults with heart failure secondary to aortic stenosis for whom surgical } \\
\text { aortic valve implantation is considered unsuitable or high risk } \\
\text { Transcatheter aortic valve implantation }\end{array}$ \\
Intervention & $\begin{array}{l}\text { Surgical aortic valve implantation } \\
\text { Medical/conservative management }\end{array}$ \\
Comparator & No intervention \\
& 1. Mortality \\
2. Major cardiovascular events \\
3. Dyspnoea \\
4. Echocardiographic criteria: ejection fraction \\
5. Length of hospital stay and readmission rates including critical care units \\
6. Quality of life \\
7. Adverse events \\
8. Cost effectiveness \\
English only \\
Randomised controlled trials from 2016 onwards \\
Types of evidence and years \\
Economic analyses from 2011 onwards \\
Exclusions
\end{tabular}


Table 2 Summary of the three randomised controlled trials and the references included in this rapid evidence review

\begin{tabular}{llllll}
\hline Patient risk group & Comparator & Trial name & References & Participants (TAVI vs comparator) & TAVI device \\
\hline Inoperable & Standard therapy & PARTNER 1B & Leon et al. (2010) [25] & $n=358$ (179 and 179) & Edwards SAPIEN \\
& & & Kapadia et al. (2015) [26] & & \\
High risk & SAVR & PARTNER 1A & Smith et al. (2011) [27] & $n=700$ (348 and 351) & Edwards SAPIEN \\
& & & Mack et al. (2015) [28] & & Medtronic CoreValve \\
& & CoreValve & Adams et al. (2014) [29] & $n=748$ (391 and 357) & \\
& & Deeb et al. (2016) [30] & & \\
& & & Gleason et al. (2016) [31] & $n=199$ (111 and 88) & \\
\hline
\end{tabular}

SAVR surgical aortic valve replacement, TAVI transcatheter aortic valve implantation

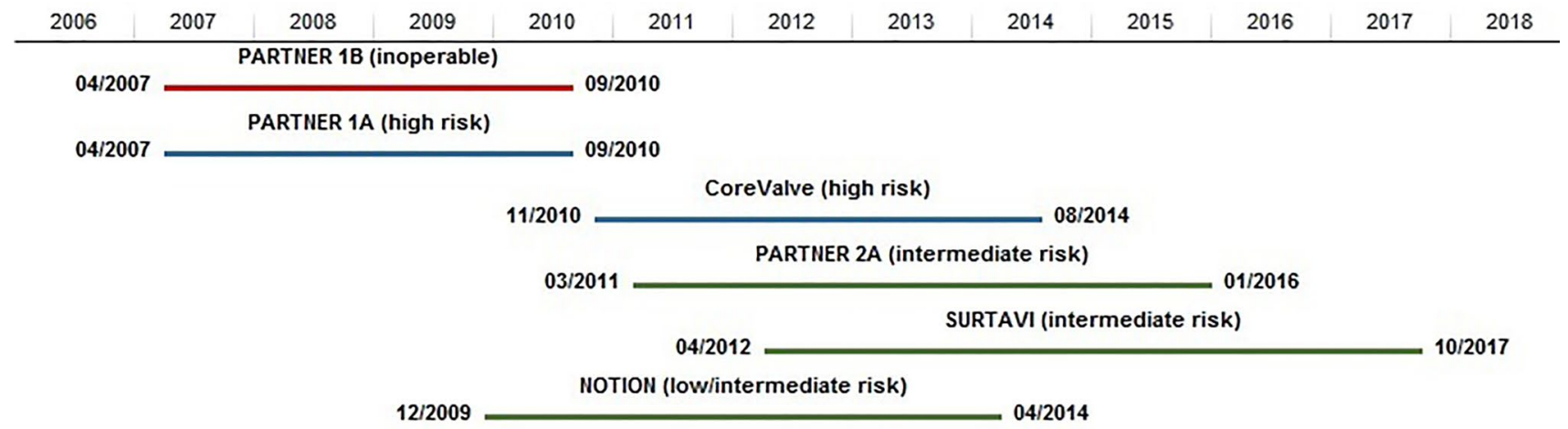

Fig. 2 Study period of all clinical trials identified form the literature search

Tables 3 and 4. All outcome measures are reported as TAVI vs comparator.

\subsubsection{Inoperable Group}

Two papers reported evidence from the PARTNER 1B RCT [25, 26]. This included patients unsuitable for surgery, defined as a probability of death or serious irreversible morbidity exceeding $50 \%$. Results suggest that TAVI has a survival advantage at 1 year [25] and 5 years [26] over medical therapy, but that TAVI carries a higher risk of vascular complications at 1 year [26].

\subsubsection{High-Risk Group}

Evidence in patients with high surgical risks was available from the PARTNER 1A and CoreValve RCTs. Two papers reported findings from the PARTNER 1A study, which included patients with a predicted risk of operative mortality of $\geq 15 \%$ and/or a STS score of $\geq 10$ [27, 28]. Three papers reported findings from the CoreValve study, which included patients with a risk of death within 30 days of surgery of $\geq 15 \%$, and a risk of death or irreversible complications of $<50 \%$ [29-31]. The PARNER 1A study reported no significant difference in mortality between TAVI and
SAVR at 30 days, 1 year or 5 years [27, 28]. However, the CoreValve trial reported significantly lower mortality for patients undergoing TAVI at 1 year [29] and at 3 years [30]. Both trials reported that TAVI carries a significantly greater risk of major vascular complications compared with SAVR at 1 year [27, 29] and 3 years [30]. At 5 years, the incidences of stroke, transient ischemic attack, myocardial infarction, endocarditis, renal failure, need for a new pacemaker or a number of hospital admissions were similar for both TAVI and SAVR [28]. An additional neurological sub-study of the CoreValve trial reported no significant difference in rates of stroke between TAVI and SAVR at 30 days, 1 year or 2 years [31].

\subsection{Cost-Effectiveness Evidence}

Freeman et al. [23] performed a retrospective cohort analysis using linked data from patients in South Wales unsuitable for SAVR. They reported significantly higher survival rates, and less frequent and shorter subsequent hospital admissions, in patients who had TAVI compared with medical treatment. They concluded that TAVI offers an additional 1.29 QALYs and calculated an ICER of $£ 10,533$ per QALY over a 5-year time horizon (2012 prices). 


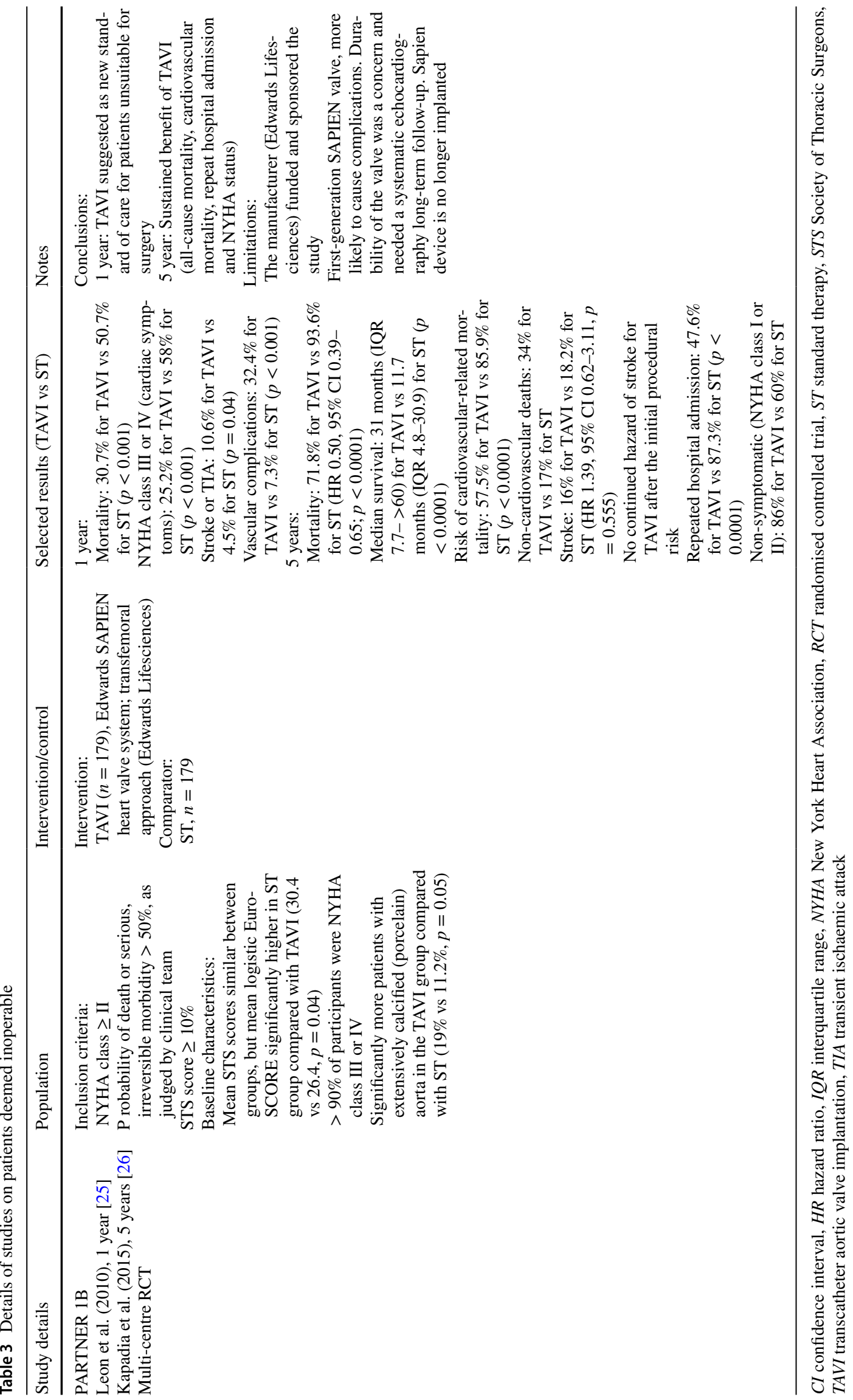




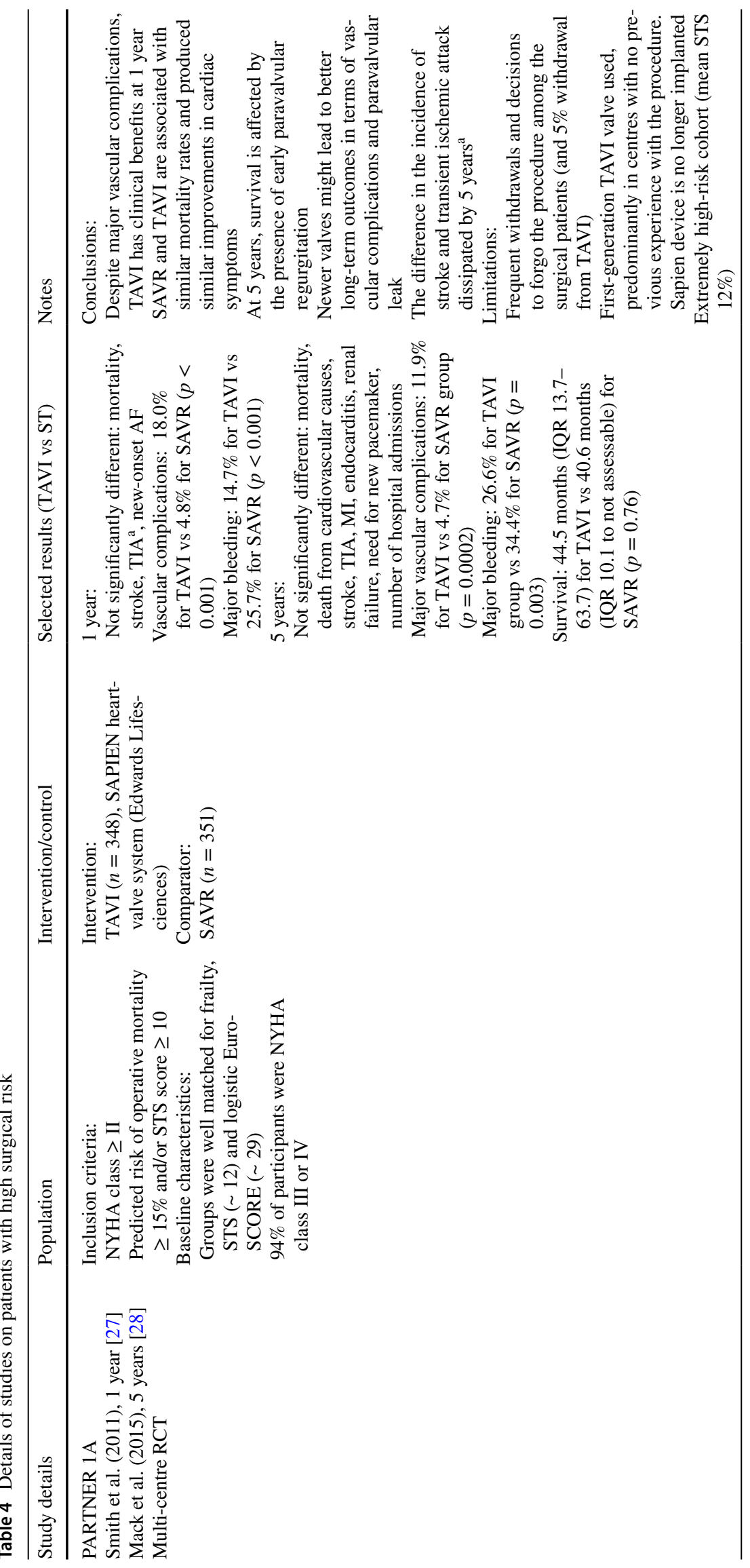




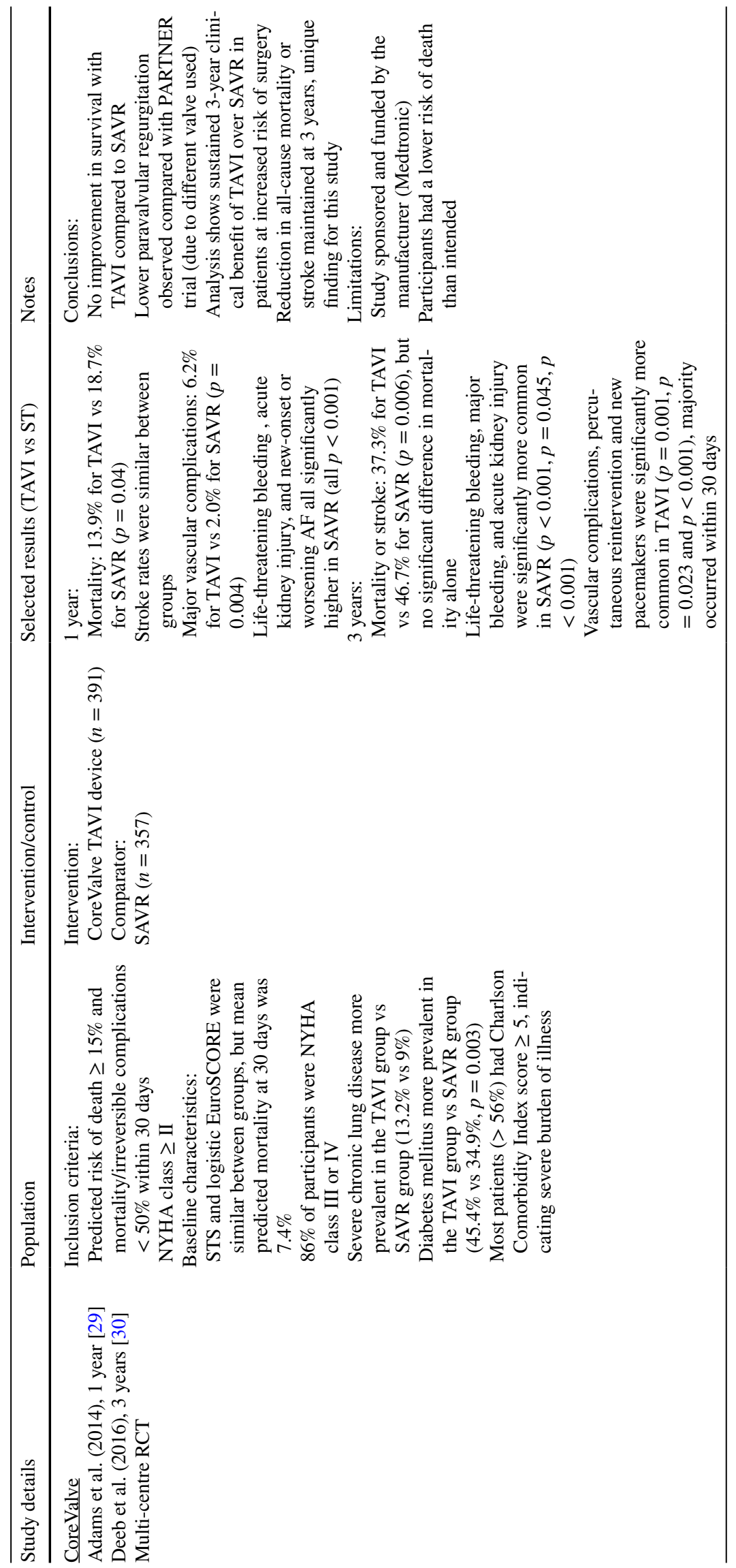




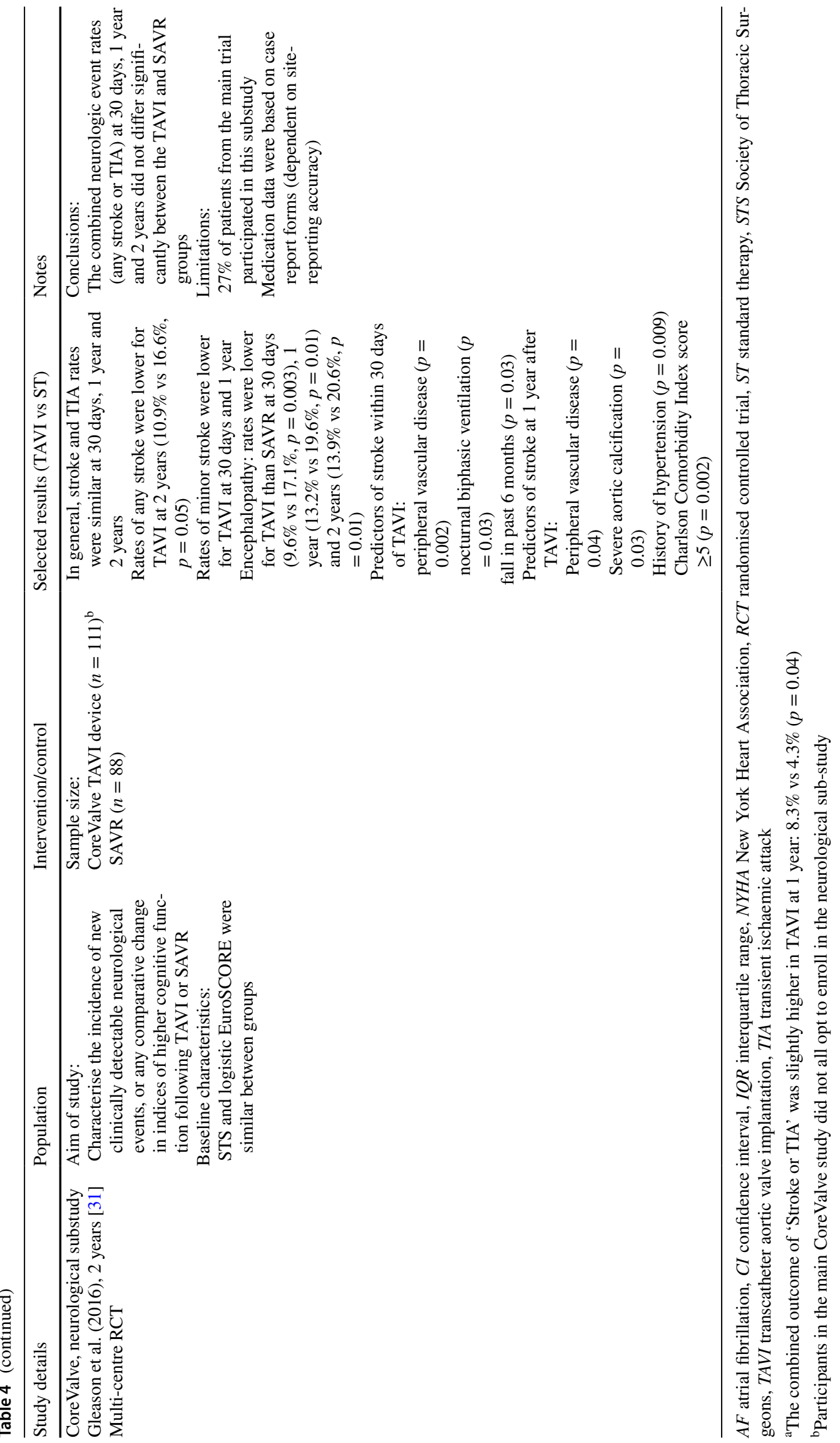


Brecker et al. [24] conducted a cost-effectiveness analysis using TAVI data from a single-arm cohort study (CoreValve ADVANCE) and outcomes for medical treatment from PARTNER B. In the high-risk subgroup, TAVI had a benefit of 1.24 QALYs and an ICER of $£ 17,718$ per QALY over 5 years (2013 prices). The model outcome was most sensitive to the cost of hospital admissions for heart failure.

\section{Updating the Commissioning Policy (CP)}

As part of the policy review, WHSSC engaged with cardiac surgeons and cardiologists from across Wales and established an EAG, with whom they shared the RER. The EAG recommended a series of changes to the population eligible to receive TAVI. The major changes to the $\mathrm{CP}$ were the inclusion of patients considered inoperable and the removal of explicit risk definitions using numeric scoring:

- Patients considered high risk or inoperable should be referred to the relevant TAVI MDT for consideration of the most appropriate aortic intervention.

- TAVI should be considered if the patient:

- has a confirmed diagnosis of severe symptomatic AS and has been discussed at the TAVI MDT; and

- is considered high risk for SAVR (owing to age, frailty and co-morbidities); or

- is deemed inoperable but the TAVI MDT concludes that significant symptomatic and/or survival benefit will be offered by TAVI, e.g. porcelain aorta.

The EAG also estimated the impact of the revision on increased activity and additional service infrastructure, which were costed by WHSSC. WHSSC estimated an increase in procedure activity across Wales of $20-30 \%$. Investment would be required to enable this: additional TAVI valves, additional consultant and clinical nurse specialist time for the MDTs, catheter laboratory staff, preprocedure and post-procedure frailty assessment, post-procedure cardiac rehabilitation and ongoing follow-up for the management of heart failure.

The EAG agreed that any revised policy should continue to exclude patients with intermediate and low surgical risk. Figure 2 shows how the evidence base had evolved from higher to lower risk patient populations at the time of the review. The intermediate-risk group is becoming increasingly important, thus emerging trial data should be kept under close observation and the TAVI policy regularly reviewed and updated.

Based on these findings, a revised $\mathrm{CP}$ was produced and issued for stakeholder consultation for 6 weeks, in line with WHSSC methodology [32]. Stakeholder feedback was incorporated into a revised and finalised $\mathrm{CP}$, with new criteria for treatment [33]. This revised $\mathrm{CP}$ was then assessed by the WHSSC Prioritisation Panel. The scoring and ranking of 23 new interventions (including TAVI) was carried out by the Panel in October 2018 .

Assessed against the four criteria (described in Sect. 1.1), the revised TAVI policy was ranked as the second highest priority considered for WHSSC funding in 2019-20. The additional investment to support implementation of the revised TAVI policy was agreed by the WHSSC Joint Committee in January 2019 and published in their ICP in March 2019 [34].

\section{Discussion and Conclusions}

We report the process for updating the $\mathrm{CP}$ for specialised services for Welsh patients using TAVI as an exemplar. A RER was conducted as part of a health technology assessment update on the use of TAVI for treating severe AS. The evidence suggests that, for inoperable and high-risk patient groups, TAVI results in better survival compared with medical therapy or SAVR respectively, although there is an increased risk of vascular complications compared with SAVR. Although TAVI is generally considered more expensive than both medical management and SAVR, the increased survival means that TAVI may be cost effective in the highest risk groups: ICERs for TAVI compared to both medical management and SAVR were mostly $<£ 24,000$ per QALY. The RER informed a revision of the WHSSC CP for TAVI, following expert review and stakeholder consultation. This expanded the eligible population, from patients with a high surgical risk, to also include patients deemed inoperable. The updated policy was published in March 2019 [33], and as a result, the number of TAVI procedures rose from 222 in 2018-2019 to 317 in 2019-2020, an increase of 45\%.

We did not conduct a traditional systematic review because of time and resource constraints. A rapid review streamlines the systematic review methodology in order to achieve useful and reliable outputs in a shorter timeframe and with fewer resources. Health technology assessment processes require decision making to be made in a timely but evidence-based manner, and pragmatism must often be balanced against strict methodological rigour in the production of CPs or guidance. Often a 'best available evidence' approach must be used. The use of relevant existing systematic reviews and other health technology assessment reports, as done here, is an appropriate means to shorten the process. This RER did not include all publications from each RCT, but did ensure inclusion and exclusion criteria, baseline characteristics, co-morbidities and key efficacy outcomes were included, with results from the longest followup period. Regular reviews between Cedar and WHSSC 
ensured that methods and results remained appropriate to the commissioners of these services.

Most of the trials included in the RER used first-generation valves (Edwards SAPIEN and Medtronic CoreValve). Technological improvements in the devices and increased clinical experience of TAVI are likely to have reduced the risks of complications and improved outcomes since these trials were conducted. This is typical of guidance on medical devices or procedures, where evolving technologies or techniques can outpace the evidence generation and review processes. Several ongoing trials were identified at the time of the RER, which may have reported in time for the next review of WHSSC CP, expected in 2022.

The surgical risk assessment scores (STS, EuroSCORE) are strongly dependent on age, and the majority of high-risk patients are elderly with co-morbidities. For these patients, improved quality of life may take precedence over increased life expectancy, and TAVI avoids the pain associated with a sternotomy and reduces the length of hospital stay compared with SAVR. However, for younger patients with mild-tomoderate disease, life expectancy may be more important and the 5-year follow-up for the majority of controlled studies may be inadequate.

The American Heart Association/American College of Cardiology guidelines for valvular heart disease were updated in December 2020 [35]. These expanded the indications for TAVI, from only patients with a prohibitive surgical risk, to include patients with a high surgical risk. For symptomatic patients of any age with severe AS and a high or prohibitive surgical risk, TAVI is recommended if predicted post-TAVI survival is $>12$ months with an acceptable quality of life. Most European and US guidelines are now therefore substantially consistent by providing or recommending TAVI in patients deemed inoperable or at high surgical risk.

Acknowledgements The authors acknowledge Dr. Susan O'Connell (Cedar, Cardiff and Vale UHB) and Dr. Helen Morgan (Cedar, Cardiff University) who contributed to the review of the manuscript and further feedback.

\section{Declarations}

Funding Cedar, a department within the Cardiff and Vale University Health Board, receives funding from WHSSC.

Conflicts of interest/Competing interests Andrew Champion, Iolo Doull and Sian Lewis are employees of WHSSC. No other conflicts of interest exist.

Ethics approval Not applicable.

Consent to participate Not applicable.

Consent for publication Not applicable.

Availability of data and material Not applicable.
Code availability Not applicable.

Authors' contributions ER, AC and LK conducted the RER, with AC, ID and SL providing oversight and liaison with WHSSC. ER and SP substantially drafted the manuscript, with a review of the text by all the authors.

Open Access This article is licensed under a Creative Commons Attribution-NonCommercial 4.0 International License, which permits any non-commercial use, sharing, adaptation, distribution and reproduction in any medium or format, as long as you give appropriate credit to the original author(s) and the source, provide a link to the Creative Commons licence, and indicate if changes were made. The images or other third party material in this article are included in the article's Creative Commons licence, unless indicated otherwise in a credit line to the material. If material is not included in the article's Creative Commons licence and your intended use is not permitted by statutory regulation or exceeds the permitted use, you will need to obtain permission directly from the copyright holder. To view a copy of this licence, visit http://creativecommons.org/licenses/by-nc/4.0/.

\section{References}

1. Dickinson M. NHS Wales specialised services strategy. 2012. https://whssc.nhs.wales/publications/strategies-and-plans/speci alised-services-strategy/. Accessed 31 Aug 2021.

2. Anderson P, Webb P, Groves S. Prioritisation of specialist health care services; not NICE, not easy but it can be done. Health Policy. 2017;121(9):978-85. https://doi.org/10.1016/j.healthpol. 2017.06.007.

3. WHSSC. An integrated commissioning plan for specialised services for Wales 2020-2023. 2020. https://whssc.nhs.wales/publi cations/integrated-commissioning-plan-2020-2023. Accessed 31 Aug 2021.

4. WHSSC. ICP 2018-2021 Annex 6 WHSSC prioritisation methodology. 2018. https://whssc.nhs.wales/publications/integratedcommissioning-plan-2018-2021/. Accessed 19 Oct 2021.

5. Kanwar A, Thaden JJ, Nkomo VT. Management of patients with aortic valve stenosis. Mayo Clin Proc. 2018;93(4):488-508. https://doi.org/10.1016/j.mayocp.2018.01.020.

6. Healthcare Improvement Scotland. Transcatheter aortic valve implantation (TAVI) for severe symptomatic aortic stenosis in adults at high surgical risk. Evidence Note 64. 2017. https://shtg. scot/our-advice/transcatheter-aortic-valve-implantation-tavi-forsevere-symptomatic-aortic-stenosis-in-adults-at-high-surgicalrisk/. Accessed 31 Aug 2021.

7. Schwarz F, Baumann P, Manthey J, et al. The effect of aortic valve replacement on survival. Circ. 1982;66(5):1105-10. https://doi. org/10.1161/01.cir.66.5.1105.

8. Dewey TM, Brown D, Ryan WH, Herbert MA, Prince SL, Mack MJ. Reliability of risk algorithms in predicting early and late operative outcomes in high-risk patients undergoing aortic valve replacement. J Thorac Cardiovasc Surg. 2008;135(1):180-7. https://doi.org/10.1016/j.jtcvs.2007.09.011.

9. WHSSC. Specialised services policy: CP58. Transcatheter aortic valve implantation (TAVI) for severe symptomatic aortic stenosis (SSAS). 2012.

10. NHS England. Clinical commissioning policy: transcatheter aortic valve implantation (TAVI) for aortic stenosis (NHSCB/A09/P/a). 2013. https://www.england.nhs.uk/wp-content/uploads/2013/04/ a09-p-a.pdf. Accessed 31 Aug 2021.

11. National Institute for Health and Care Excellence. Transcatheter aortic valve implantation for aortic stenosis. Interventional 
procedure guidance (IPG586). 2017. Available from: https://www. nice.org.uk/guidance/ipg586. Accessed 31 Aug 2021.

12. National Institute for Health and Care Excellence. Transcatheter aortic valve implantation for aortic stenosis. Interventional procedure guidance (IPG421). 2012.

13. National Institute for Health and Care Excellence. Acute heart failure: diagnosis and management. Clinical guideline (CG187). 2014. https://www.nice.org.uk/guidance/cg187. Accessed 31 Aug 2021.

14. Murphy A, Fenwick E, Toff WD, Neilson MP, Berry C, Uren $\mathrm{N}$, et al. Transcatheter aortic valve implantation for severe aortic stenosis: the cost-effectiveness case for inoperable patients in the United Kingdom. Int $\mathbf{J}$ Technol Assess Health Care. 2013;29(1):12-9. https://doi.org/10.1017/S0266462312000670.

15. Orlando R, Pennant M, Rooney S, Khogali S, Bayliss S, Hassan A, et al. Cost-effectiveness of transcatheter aortic valve implantation (TAVI) for aortic stenosis in patients who are high risk or contraindicated for surgery: a model-based economic evaluation. Health Technol Assess. 2013;17(33):1-86. https://doi.org/10. 3310/hta17330.

16. Watt M, Mealing S, Eaton J, Piazza N, Moat N, Brasseur P, et al. Cost-effectiveness of transcatheter aortic valve replacement in patients ineligible for conventional aortic valve replacement. Heart. 2012;98(5):370-6. https://doi.org/10.1136/heart jnl-2011-300444.

17. Fairbairn TA, Meads DM, Hulme C, Mather AN, Plein S, Blackman DJ, et al. The cost-effectiveness of transcatheter aortic valve implantation versus surgical aortic valve replacement in patients with severe aortic stenosis at high operative risk. Heart. 2013;99(13):914-20. https://doi.org/10.1136/heart jnl-2013-303722.

18. Scottish Health Technologies Group. Transcatheter aortic valve implantation (TAVI) for severe symptomatic aortic stenosis in adults at high surgical risk: advice statement 001-17. 2017. https:// shtg.scot/our-advice/transcatheter-aortic-valve-implantation-tavifor-severe-symptomatic-aortic-stenosis-in-adults-at-high-surgicalrisk/. Accessed 31 Aug 2021.

19. Baumgartner H, Falk V, Bax JJ, De Bonis M, Hamm C, Holm PJ, ESC Scientific Document Group, et al. 2017 ESC/EACTS guidelines for the management of valvular heart disease. Eur Heart J. 2017;38(36):2739-91. https://doi.org/10.1093/eurheartj/ehx391.

20. Nishimura RA, Otto CM, Bonow RO, Carabello BA, Erwin JP, Guyton RA, et al. 2014 AHA/ACC guideline for the management of patients with valvular heart disease: a report of the American College of Cardiology/American Heart Association Task Force on Practice Guidelines. J Am Coll Cardiol. 2014;63(22):e57-185. https://doi.org/10.1161/CIR.0000000000000031.

21. Tricco AC, Antony J, Zarin W, Strifler L, Ghassemi M, Ivory $\mathrm{J}$, et al. A scoping review of rapid review methods. BMC Med. 2015;13(1):1-5. https://doi.org/10.1186/s12916-015-0465-6.

22. National Institute for Health and Care Excellence. Interventional procedure overview of transcatheter aortic valve implantation for aortic stenosis. 2017. https://www.nice.org.uk/guidance/ipg586/ evidence/overview-final-pdf-4541961565. Accessed 31 Aug 2021.

23. Freeman PM, Protty MB, Aldalati O, Lacey A, King W, Anderson RA, et al. Severe symptomatic aortic stenosis: medical therapy and transcatheter aortic valve implantation (TAVI): a real-world retrospective cohort analysis of outcomes and cost-effectiveness using national data. Open Heart. 2016;3(1): e000414. https://doi. org/10.1136/openhrt-2016-000414.
24. Brecker S, Mealing S, Padhiar A, Eaton J, Sculpher M, Busca R, et al. Cost-utility of transcatheter aortic valve implantation for inoperable patients with severe aortic stenosis treated by medical management: a UK cost-utility analysis based on patient-level data from the ADVANCE study. Open Heart. 2014;1(1): e000155. https://doi.org/10.1136/openhrt-2014-000155.

25. Leon MB, Smith CR, Mack M, Miller DC, Moses JW, Svensson LG, et al. Transcatheter aortic-valve implantation for aortic stenosis in patients who cannot undergo surgery. N Engl J Med. 2010;363(17):1597-607. https://doi.org/10.1056/nejmoa1008232.

26. Kapadia SR, Leon MB, Makkar RR, Tuzcu EM, Svensson LG, Kodali $\mathrm{S}$, et al. 5-year outcomes of transcatheter aortic valve replacement compared with standard treatment for patients with inoperable aortic stenosis (PARTNER 1): a randomised controlled trial. Lancet. 2015;385(9986):2485-91. https://doi.org/10.1016/ S0140-6736(15)60290-2.

27. Smith CR, Leon MB, Mack MJ, Miller DC, Moses JW, Svensson LG, et al. Transcatheter versus surgical aortic-valve replacement in high-risk patients. N Eng J Med. 2011;364(23):2187-98. https://doi.org/10.1056/NEJMoa1103510.

28. Mack MJ, Leon MB, Smith CR, Miller DC, Moses JW, Tuzcu EM, et al. 5-year outcomes of transcatheter aortic valve replacement or surgical aortic valve replacement for high surgical risk patients with aortic stenosis (PARTNER 1): a randomised controlled trial. Lancet. 2015;385(9986):2477-84. https://doi.org/10.1016/S01406736(15)60308-7.

29. Adams DH, Popma JJ, Reardon MJ, Yakubov SJ, Coselli JS, Deeb GM, et al. Transcatheter aortic-valve replacement with a self-expanding prosthesis. N Eng J Med. 2014;370(19):1790-8. https://doi.org/10.1056/NEJMoa1400590.

30. Deeb GM, Reardon MJ, Chetcuti S, Patel HJ, Grossman PM, Yakubov SJ, et al. 3-year outcomes in high-risk patients who underwent surgical or transcatheter aortic valve replacement. J Am Coll Cardiol. 2016;67(22):2565-74. https://doi.org/10.1016/j. jacc.2016.03.506.

31. Gleason TG, Schindler JT, Adams DH, Reardon MJ, Kleiman NS, Caplan LR, et al. The risk and extent of neurologic events are equivalent for high-risk patients treated with transcatheter or surgical aortic valve replacement. J Thorac Cardiovasc Surg. 2016;152(1):85-96. https://doi.org/10.1016/j.jtcvs.2016.02.073.

32. WHSSC. Consultation guide for commissioning policies and service specifications (054b). 2015. https://whssc.nhs.wales/publi cations/corporate-policies-and-procedures/corp054b-consultati on-policy/. Accessed 31 Aug 2021.

33. WHSSC. Specialised services commissioning policy: CP58. Trans-catheter aortic valve implantation (TAVI) for severe symptomatic aortic stenosis (SSAS). 2019. https://whssc.nhs.wales/ commissioning/whssc-policies/cardiac/cp58-trans-catheter-aortic-valve-implantation-tavi-for-severe-symptomatic-aortic-steno sis-ssas-pdf/. Accessed 31 Aug 2021.

34. WHSSC. An integrated commissioning plan for specialised services for Wales 2019 - 2022. 2019. https://whssc.nhs.wales/publi cations/integrated-commissioning-plan-2019-2022/. Accessed 31 Aug 2021.

35. Otto CM, Nishimura RA, Bonow RO, Carabello BA, Erwin JP III, Gentile F, et al. 2020 ACC/AHA guideline for the management of patients with valvular heart disease: a report of the American College of Cardiology/American Heart Association Joint Committee on Clinical Practice Guidelines. Circulation. 2021;143:e72-227. https://doi.org/10.1161/CIR.0000000000000923. 